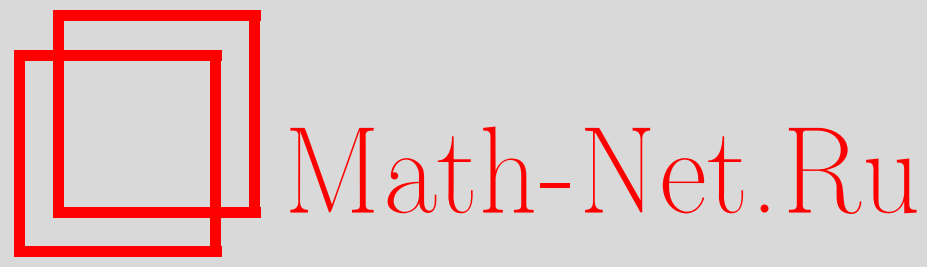

А. Л. Крюкова, Об округлениях в топологических частично упорядоченных пространствах, Функи. анализ и его прил., 2012, том 46, выпуск 1, 83-87

DOI: https://doi.org/10.4213/faa3063

Использование Общероссийского математического портала MathNet.Ru подразумевает, что вы прочитали и согласны с пользовательским соглашением

http://www . mathnet.ru/rus/agreement

Параметры загрузки:

IP : 54.80 .97 .219

26 апреля 2023 г., $11: 28: 42$

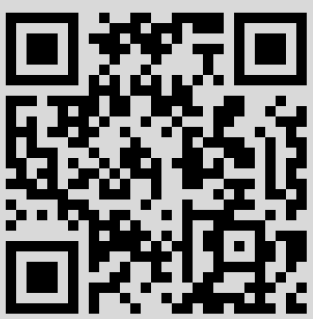




\title{
ЛитерАТУРА
}

[1] Ю. Г. Борисович, Б. Д. Гельман, А. Д. Мышкис, В. В. Обуховский, Введение в теорию многозначных отображений, КомКнига (URSS), M, 2005. [2] J. Saint Raymond, C. R. Acad. Sci. Paris, 298 (1984), 71-74. [3] Б. Д. Гельман, Функц. анализ и его прил., 42:3 (2008), 78-81.

Воронежский государственный университет

Поступило в редакцию e-mail: gelman@func.vsu.ru

19 мая 2010 г.

УДК 517.984

\section{Об округлениях в топологических частично упорядоченных пространствах}

\author{
(c) 2012. А. Л. КрюковА
}

1. Введение. В практике компьютерных вычислений одна из наиболее часто встречающихся операций - это округление (промежуточных) результатов. Истоки аксиоматической теории округлений содержатся в работе Кулиша [4], в которой он определил их как отображения линейно упорядоченного множества в его подмножество, удовлетворяющие некоторым естественным требованиям (аксиомам). Понятие интервального округления было сформулировано и систематически изучалось в работах [2], [3]. В данной работе интервальные округления изучаются в рамках общей теории топологических частично упорядоченных пространств. Мы устанавливаем некоторые общие свойства округлений, (частично) характеризуем их в классе изотонных отображений, изучаем способы их описания и, в частности, для интервальных округлений строим функциональную модель, позволяющую во многих случаях сводить изучение свойств округлений к чисто аналитическим вопросам.

2. Округления и их неподвижные точки. Напомним, что частично упорядоченное пространство называется решеткой, если любые два его элемента обладают точной нижней и точной верхней гранями. Решетка называется полной (условно полной), если точную верхнюю и точную нижнюю грани имеет каждое (соответственно каждое ограниченное) ее подмножество.

Мы будем называть упорядоченное пространство условно $l$-полным, если любое ограниченное снизу множество имеет точную нижнюю грань.

Для любого упорядоченного пространства $(X, \leqslant)$ обозначим через $I E(X)$ совокупность всех его отображений $\varphi$ в себя, для которых 1$) x \leqslant \varphi(x)$ и 2) $\varphi\left(x_{1}\right) \leqslant \varphi\left(x_{2}\right)$, если $x_{1} \leqslant x_{2}$. Отображение $\varphi \in I E(X)$ называется (верхним) округлением (или замыканием [1]), если оно идемпотентно, т. е. удовлетворяет условию 3) $\varphi(\varphi(x))=\varphi(x)$.

Округления $\varphi$ и $\psi$ в пространствах $(X, \leqslant)$ и $(Y, \leqslant)$ назовем эквивалентными, если существует сохраняющая порядок биекция $f: X \rightarrow Y$, такая, что

$$
f(\varphi(x))=\psi(f(x))
$$

для любого $x \in X$. 
Множество точек $x \in X$, для которых $\varphi(x)=x$, будем называть множеством неподвижных точек округления $\varphi$ и обозначать через $E_{\varphi}$.

Теорема 1. Округления $\varphi$ и н на условно $l$-полных пространствах $X u$ $Y$ соответственно эквивалентны, если существует возрастающая биекиия пространства $X$ на $Y$, отображсающая $E_{\varphi}$ на $E_{\psi}$.

Пусть $G$ - некоторая группа автоморфизмов пространства $(X, \leqslant)$. Отображение $\varphi$ называется ковариантным относительно $G$, если оно коммутирует с элементами из $G$, т. е. если $\varphi(T(x))=T(\varphi(x))$ для всех $T \in G$. В частности, можно говорить о ковариантных округлениях.

Следствие 2. Округление $\varphi$ ковариантно относительно некоторой группы автоморфизмов тогда и только тогда, когда $E_{\varphi}$ инвариантно относительно этой группы.

Округление $\varphi$ называется идемпотентно аддитивным, если для любых двух элементов $x, y \in X$, таких, что $x \vee y$ существует,

$$
\varphi(x \vee y)=\varphi(x) \vee \varphi(y) .
$$

Следствие 3. Пусть условно l-полное пространство $X$ таково, что у любых двух элементов существует точная верхняя грань. Округление $\varphi$ на $X$ идемпотентно аддитивно тогда и толъко тогда, когда $x \vee y \in E_{\varphi}$ для любых $x, y \in E_{\varphi}$.

Легко видеть, что множество $I E(X)$ является полугруппой относительно композиции, а множество $O(X)$ всех округлений - это множество всех идемпотентных элементов этой полугруппы. Следовательно, композиция двух округлений является округлением тогда и только тогда, когда эти округления перестановочны. Поэтому для изучения подполугрупп в $I E(X)$, состоящих из округлений, важно знать, какие округления перестановочны.

Следствие 4. Два округления $\varphi, \psi$ перестановочны тогда и только тогда, когда $\varphi\left(E_{\psi}\right) \subset E_{\psi} u \psi\left(E_{\varphi}\right) \subset E_{\varphi}$.

Пусть $X$ - выпуклое подмножество линейного вещественного пространства $\mathscr{X}$, а упорядочение задается выпуклым конусом $\mathscr{K}$. В этих условиях $I E(X)$ обладает выпуклой структурой, т.е. является выпуклым подмножеством линейного пространства всех отображений из $X$ в $\mathscr{X}$.

Теорема 5. Всякое округление в $(X, \leqslant \mathscr{K})$ является крайней точкой в $I E(X)$.

3. Непрерывность снизу. Пусть в упорядоченном множестве $(X, \leqslant)$ задана топология. Одним из наиболее естественных условий согласованности ее с порядком является условие

(i) график порядка $\Gamma=\{(x, y): x \leqslant y\}$ - замкнутое подмножество в $X \times X$.

Пространство $(X, \leqslant)$ называется интервально компактным, если выполнено условие

(k) Для любых $x, y \in X$ множество $I(x, y)=\{z: x \leqslant z \leqslant y\}$ является компактным.

Рассмотрим теперь условие, которое, будучи значительно сильнее условия (i), тем не менее, выполняется во многих важных примерах:

(ii) график порядка является замыканием своей внутренности.

Внутренность множества $\Gamma$ определяет на $X$ отношение $\prec$ «строгого» порядка (т.е. транзитивное, но не рефлексивное), порожденное отношением $\leqslant$ : 
$x \prec y$, если $(x, y) \in \operatorname{int}(\Gamma)$. Другими словами, $x \prec y$, если существуют окрестности $U \ni x, V \ni y$, такие, что $x^{\prime} \leqslant y^{\prime}$ при любых $x^{\prime} \in U, y^{\prime} \in V$.

Удобно также «детализировать» отношение $\prec$, полагая $x \stackrel{1}{\prec} y$, если существует окрестность $U \ni x$, такая, что $x^{\prime} \leqslant y$ для всех $x^{\prime} \in U$, и, аналогично, $x \stackrel{2}{\prec} y$, если существует окрестность $V \ni y$, такая, что $x \leqslant y^{\prime}$ для всех $y^{\prime} \in V$. В ряде примеров (например, для аддитивно инвариантных порядков в линейных пространствах) эти отношения совпадают. Однако в общем случае приходится отдельно формулировать два условия:

(iii) для любого $y \in X$ множество всех $x$, таких, что $x \leqslant y$, является замыканием множества всех $x$, таких, что $x \stackrel{1}{\prec} y$;

(iv) для любого $x \in X$ множество всех $y$, таких, что $x \leqslant y$, является замыканием множества всех $y$, таких, что $x \stackrel{2}{\prec} y$.

Будем говорить, что $(X, \leqslant)$ является $T$-полурешеткой, если для любых двух элементов существует точная верхняя грань, для любого ограниченного сверху (снизу) подмножества существует точная верхняя (точная нижняя) грань и выполнены условия (i), (iii), (iv) и (k).

Примерами могут служить банаховы решетки, упорядоченные телесным конусом, а также их замкнутые выпуклые подрешетки. Другой класс примеров условно полные решетки с порядковой топологией.

Естественный и включающий в себя все наиболее важные примеры класс округлений в топологических упорядоченных пространствах составляют непрерывные снизу, или $l$-непрерывные, округления, т. е. такие округления $\psi$, что $\psi\left(\lim x_{n}\right)=\lim \psi\left(x_{n}\right)$ для любой возрастающей сходящейся последовательности $\left\{x_{n}\right\}$.

Теорема 6. Округление на T-полурешетке $(X, \leqslant)$ l-непрерывно тогда $u$ только тогда, когда множество $E=E_{\varphi}$ всех его неподвижнных точек замкнуто.

Если топология в $T$-полурешетке $X$ задается равномерной структурой (как в случае линейно-топологических решеток или метрических решеток), то в $O(X)$ имеется топология равномерной сходимости на компактах, причем эта топология также равномерна. Возникает вопрос о полноте пространства округлений.

В постановке, не учитывающей топологические свойства пространства, ответ на него отрицателен уже в случае, когда $(X, \leqslant)$ - прямая с обычным порядком. Однако ситуация меняется, если ограничиться рассмотрением $l$-непрерывных округлений.

Теорема 7. Пространство $F_{0}$ всех l-непрерывных округлений на метрической Т-полурешетке полно.

4. Округления на прямой. Рассмотрим в качестве исходного пространства $(X, \leqslant)$ вещественную прямую $\mathbb{R}$ с обычным порядком и топологией.

В рассматриваемом случае теорема 5 допускает обращение:

Теорема 8. Пусть Я - множество всех непрерывных слева неубывающих функиий $f$ на $\mathbb{R}$, удовлетворяющих условию $f(t) \geqslant t$. Функиия $f \in \mathscr{F}$ является крайней точкой выпуклого множества Я્F тогда и только тогда, когда $f(f(x))=f(x)$.

Пусть $(X, \leqslant)$ - упорядоченное пространство с метрикой $\rho$. 
Будем говорить, что класс всех округлений на $(X, \leqslant)$ устойчив по ХайерсуУламу в классе $\mathscr{F} \subset I E(X)$ (см., например, [6]), если любое «почти идемпотентное» отображение из $\mathscr{F}$ близко к идемпотентному. Точная формулировка: для любого $\varepsilon>0$ найдется $\delta>0$, такое, что если $f \in \mathscr{F}$ и $\rho(f(f(x)), f(x))<\delta$ для всех $x$, то $\rho(f(x), \varphi(x))<\varepsilon$ для всех $x$, где $\varphi$ - некоторое округление.

Теорема 9. Класс всех $l$-непрерывных округлений на прямой устойчив по Хайерсу-Уламу в классе полунепрерывных отображений из $I E(\mathbb{R})$.

Будем говорить, что два подмножества прямой $M_{1}$ и $M_{2}$ перемежалотся, если из того, что между точками $x \in M_{1}, y \in M_{2}$ нет точек, принадлежащих $M_{1} \cup M_{2}$, следует, что хотя бы одна из точек $x, y$ принадлежит $M_{1} \cap M_{2}$.

Теорема 10. Пусть $\varphi, \psi$ суть l-непрерывные верхние округления на $\mathbb{R}$. Для того чтобы они коммутировали, необходимо и достаточно, чтобы множества $E_{\varphi}$ и $E_{\psi}$ перемежались.

5. Интервальные округления. Процедура интервализации (см. [5]) сопоставляет любому упорядоченному пространству $(X, \leqslant)$ новое упорядоченное пространство $\operatorname{Inter}(X)$, состоящее из всех пар $(x, y) \in X \times X$, таких, что $x \leqslant y$. Порядок на $\operatorname{Inter}(X)$ вводится правилом

$$
\left(x_{1}, y_{1}\right) \leqslant\left(x_{2}, y_{2}\right) \text {, если } x_{2} \leqslant x_{1}, y_{1} \leqslant y_{2} .
$$

Мы будем записывать элементы из $\operatorname{Inter}(X)$ в форме $[x, y]$, вместо $(x, y)$.

Предложение 11. Если $(X, \leqslant)$ есть T-решетка, то $\operatorname{Inter}(X)$ является Т-полурешеткой.

Округления на упорядоченном пространстве $\operatorname{Inter}(X)$ называются интервальными округлениями на $(X, \leqslant)$.

$\mathrm{B}$ частности, $\operatorname{Inter}(\mathbb{R})$ - это $T$-полурешетка замкнутых интервалов на прямой, причем порядок совпадает с теоретико-множественным включением. Естественно отождествлять интервал $[a, b]$ с точкой $(a, b)$ координатной плоскости и в этом смысле говорить, например, о проекциях какого-то множества интервалов.

Мы получим описание интервальных округлений на прямой в терминах функциональной модели.

Теорема 12. l-непрерывное интервальное округление задается выбором замкнутых подмножеств прямой $A$ и $B$, неограниченных снизу и сверху соответственно, функиии $\alpha: A \rightarrow(-\infty,+\infty]$, полунепрерывной сверху, и функиии $\beta: B \rightarrow[-\infty,+\infty)$, полунепрерывной снизу, таких, что выполнены условия

(i) функция $\alpha$ (функция $\beta$ ) является неограниченной сверху (соответственно снизу) на любом множестве вида $\{x \in A: x<a\}$ (соответственно $\{y \in B: y>b\})$;

(ii) $\alpha(x) \geqslant x, \beta(y) \leqslant y$;

(iii) $\alpha(\beta(y)) \geqslant y, \beta(\alpha(x)) \leqslant x$.

Множество неподвижных точек этого округления состоит из всех точек $(x, y) \in A \times B$, для которых $y \leqslant \alpha(x), x \geqslant \beta(y)$.

Набор $\chi=(A, B, \alpha, \beta)$, соответствующий округлению $\varphi$, называется его функииональной моделью.

В терминах функциональной модели удобно характеризовать важные специальные классы округлений. Укажем, например, как выделяется в этих терминах класс идемпотентно аддитивных округлений. 
Предложение 13. Пусть $\varphi$ есть l-непрерывное интервальное округление и $(A, B, \alpha, \beta)$ - его функииональная модель. Для того чтобы $\varphi$ было идемпотентно аддитивным, необходимо и достаточно, чтобы функиии $\alpha$ и $\beta$ были убывающими.

Условие непрерывности позволяет значительно упростить функциональную модель.

Следствие 14. Непрерывное интервальное округление $\varphi$ можно задавать условием

$$
E_{\varphi}=\{(x, y): y \geqslant \gamma(x), x \leqslant \delta(y)\},
$$

выбирая произвольные числа $a, b$ и непрерывные невозрастающие функиии $\gamma, \delta$ на $(-\infty, a])$ u $[b,+\infty)$, удовлетворяющие условиям

(a) $\delta(\gamma(x)) \geqslant x, \gamma(\delta(y)) \leqslant y$;

(b) $\lim _{x \rightarrow-\infty} \gamma(x)=+\infty, \lim _{y \rightarrow+\infty} \delta(y)=-\infty$.

Автор выражает искреннюю признательность Т. Э. Каминскому за постановку задач и полезные обсуждения.

\title{
ЛитеРАТУРА
}

[1] Г. Биркгоф, Теория структур, ИЛ, М., 1952. [2] Т. E. Kaminsky, V. Kreinovich, Notes on intuitionistic fuzzy sets, 4:3 (1998), 57-64. [3] Т. Э. Каминский, в кн.: Исследования по математическому анализу и методике преподавания математики, Русь, Вологда, 2000, 23-36. [4] U. Kulisch, Numer. Math., 18:1 (1971), 1-17. [5] G. L. Litvinov, The Maslov dequantization, idempotent and tropical mathematics: A brief introduction, http://arxiv.org/abs/math/0507014v1. [6] Е. В. Шульман, J. London Math. Soc. (2), 54:1 (1996), 111-120.

Вологодский государственный педагогический университет e-mail: kryukovanastya@yahoo.com

Поступило в редакцию 18 января 2010 г.

\section{Сингулярные интегральные операторы в весовых пространствах Лебега с переменными показателями на сложных карлесоновских кривых}

\author{
(c) 2012. В. С. РАБинович, С. Г. Самко
}

Памяти профессора И. Б. Симоненко посвящается

1. Введение. В работе [15] И. Б. Симоненко впервые ввел локальный метод исследования фредгольмовости одномерных и многомерных сингулярных интегральных операторов (СИО). Посредством этого метода исследование фредгольмовости СИО $A=a I+b S_{\Gamma}$ с непрерывными коэффициентами $a$ и $b$, действующих в $L^{p}(\Gamma), 1<p<\infty$, на кривых Ляпунова $Г$, сводилось к исследованию локальных представителей оператора $A$ в каждой точке $t_{0} \in \Gamma$, являющихся простейшими операторами типа $A_{t_{0}}=a\left(t_{0}\right) I+b\left(t_{0}\right) S_{\mathbb{R}}$, действуюшими в $L^{p}(\mathbb{R})$. Этот метод был применен в [14] к СИО с кусочно непрерывными коэффициентами, действующими в пространствах $L^{p}(\Gamma, w)$ со степенным весом 